\title{
Exploration of Novel Ordering Mechanism in Titanium Alloys Using Atom Probe Tomography and Aberration-corrected Scanning Transmission Electron Microscopy
}

\author{
Yufeng Zheng ${ }^{1}$, Stoichko Antonov ${ }^{2}$ and Hamish Fraser ${ }^{3}$ \\ ${ }^{1}$ University of Nevada Reno, Reno, Nevada, United States, ${ }^{2}$ Max-Planck-Intitut fur Eisenforschung \\ GmbH, Dusseldorf, Nordrhein-Westfalen, Germany, ${ }^{3}$ The Ohio State University, Columbus, Ohio, United \\ States
}

Microstructural evolution and phase transformations in titanium alloys are closely related to the beta phase stability. With the development of advanced characterization techniques, e.g. atom probe tomography (APT) and aberration-corrected scanning transmission electron microscopy (STEM), various novel nanoscaled structural and/or compositional instabilities in beta phase have been explored in titanium alloys recently, including athermal and isothermal omega phase [1], incommensurate nano-domains [2], O' phase [3] and nano-scaled solute segregation via spinodal decomposition [4]. For example, in our recent studies, the structure and composition of nano-scaled isothermal omega phase has been systematically explored in a metastable beta titanium alloy, Ti-5Al-5Mo-5V-3Cr (Ti-5553, wt.\%), through coupling APT and aberration-corrected STEM data [1]. Via these atomic resolution characterizations, it has been clearly shown that the hexagonal structure of nano-scaled isothermal omega phase is formed via the collapse of every two of three adjacent $\{111\}$ plane where solutes including $\mathrm{Al}, \mathrm{Mo}, \mathrm{V}$ and $\mathrm{Cr}$ are all rejected from the isothermal omega phase into the beta phase by diffusion. In this presentation, the latest investigation of novel nano-scaled ordered orthorhombic structure phase in titanium alloy, named as O" phase, as studied using APT, transmission electron microscopy (TEM) and aberration-corrected STEM will be introduced [5-6].

In the first part of this work, novel O" phase was investigated using ex-situ TEM (FEI CM200) and aberration-corrected STEM (FEI Titan ${ }^{\mathrm{TM}}$ 80-300) in Ti-5553 [5]. For the first time, the nano-scaled particles of ellipsoidal morphology and ordered orthorhombic structure were observed in the aged Ti5553, shown in Fig. 1(a-b) [5]. The structure of the O" phase was characterized using Z-contrast HAADFSTEM imaging, revealing ordering of every third $\{011\}$ beta planes by the periodic intensity change of the atom columns, shown in the Fig. 1(c) [6].

The second part of the work to be presented focuses on the exploration of the compositional information of the O" phase using APT (LEAP 5000XR) [6]. For the first time, conclusive experimental evidence showing that the $\mathrm{Al}$ segregation plays an important role in the nano-scaled ordering is provided. Fig. 2(a) shows a representative 3D reconstruction where only $\mathrm{Ti}$ ions as well as an 85 at.\% $\mathrm{Ti}$ and 6.5 at. $\% \mathrm{Al}$ isoconcentration surfaces are shown, revealing the presence of nano-scaled O" phase (green colored particles) together with nano-scaled isothermal omega phase (red colored particles). The compositional profile in Fig. 2(d) across the beta/O" phase interface shows that Al slightly segregates into $\mathrm{O}$ " phase compared with the composition of isothermal omega phase (although still lower than in the parent beta phase). During heating, significant amounts of $\mathrm{V}$, Mo and $\mathrm{Cr}$ together with $\mathrm{Al}$ partition to the beta phase from the incipient nano-scaled domains, generating the heating-induced ordered orthorhombic structure of O" phase [6]. 


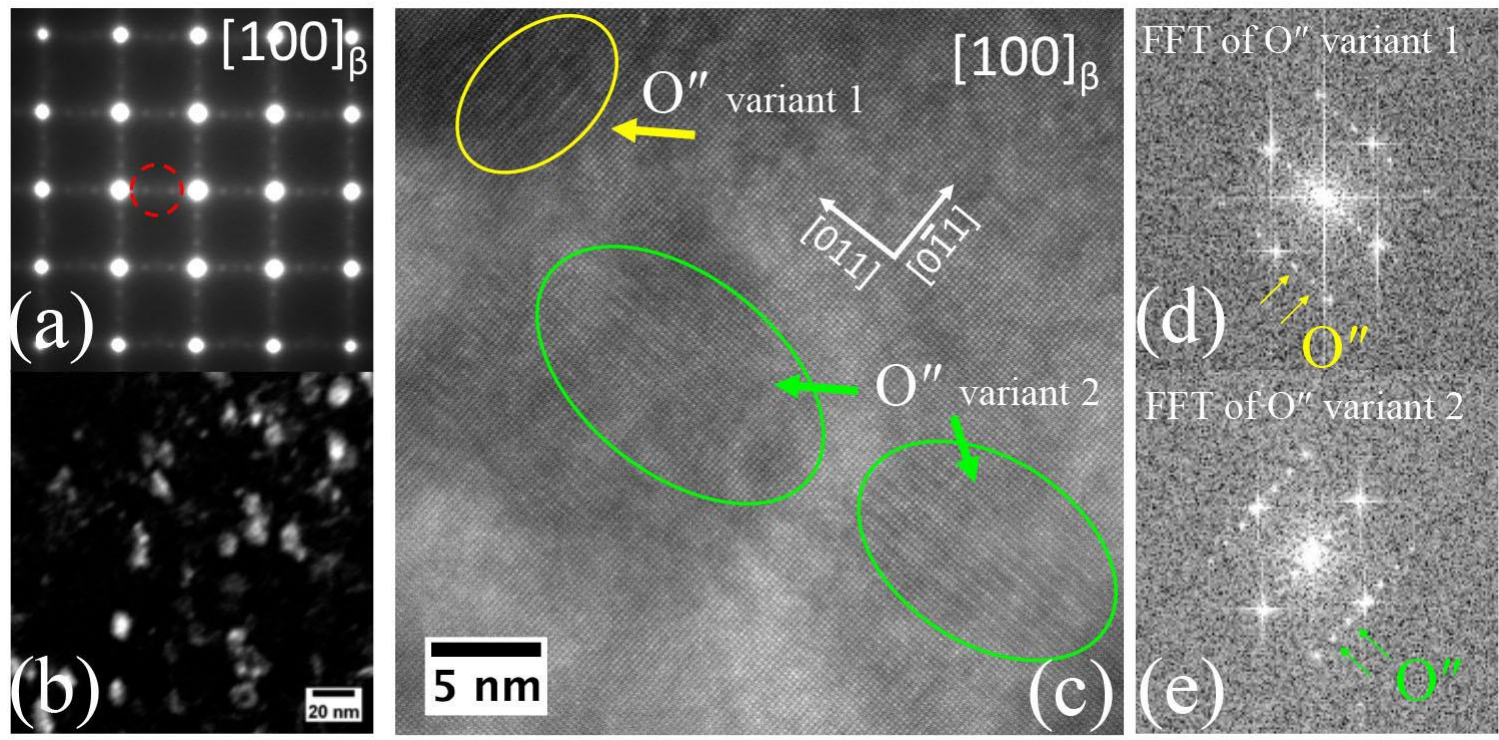

Figure 1. TEM and aberration-corrected STEM results showing the nano-scaled O" phase particles: (a) Selected area diffraction pattern and (b) corresponding dark field image showing the morphology of O" phase particles [5]; (c) HAADF-STEM image and corresponding Fast Fourier Transformations showing the ordered structure of $\mathrm{O}$ " phase [6].

(a)
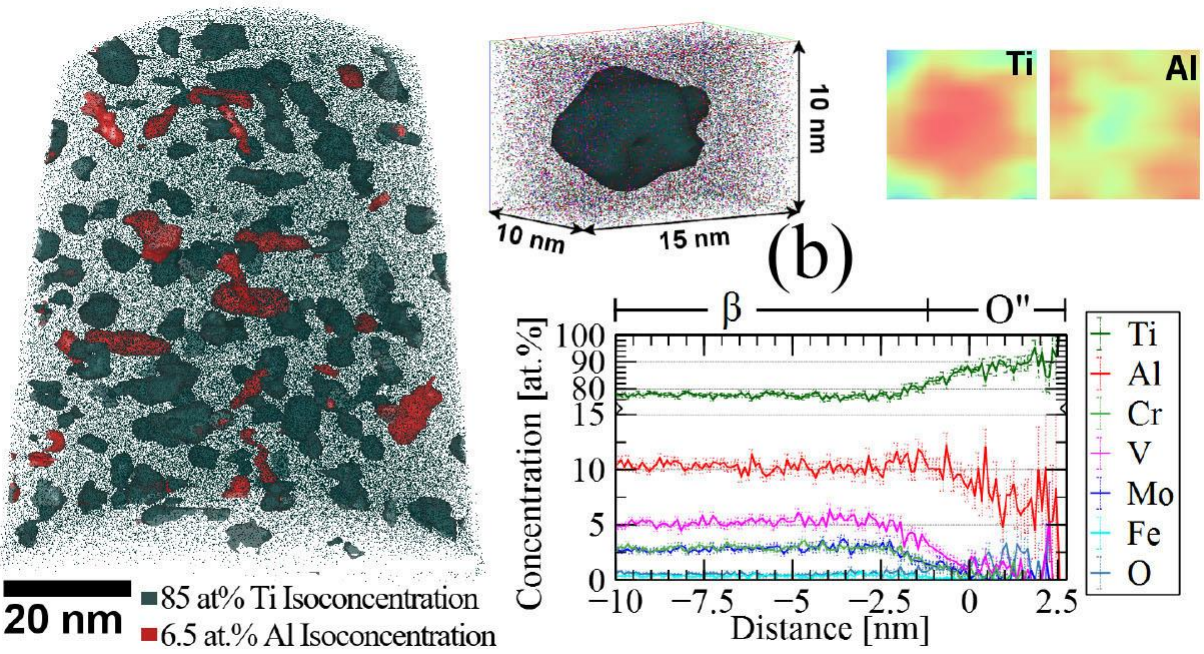

(c)

Figure 2. APT results showing nano-scaled $\mathrm{O}^{2}$ phase particles: (a) 3D reconstruction showing the morphology of O" phase particles (green) and isothermal omega phase particles (red); (b-c) selected $3 \mathrm{D}$ reconstruction of an $\mathrm{O}$ " phase particle and 2D composition slices from that particle; (d) composition profile across beta/O" interface showing the composition of $\mathrm{O}$ " phase [6].

\section{References}

[1] Y. Zheng et al., Acta Materialia, 103 (2016) 850-858.

[2] Y. Zheng et al., Scripta Materialia, 154 (2018) 220-224.

[3] Y. Zheng et al., Scripta Materialia, 116 (2016) 49-52.

[4] Y. Hao et al., Journal of Materials Science \& Technology, 32 (8) (2016) 705-709.

[5] Y. Zheng et al., Scripta Materialia, 113 (2016) 202-205.

[6] Y. Zheng et al., Scripta Materialia, 176 (2020) 7-11. 